\title{
Rapid determination of aristolochic acids I and II in herbal products and biological samples by ultra-high-pressure liquid chromatography-tandem mass spectrometry
}

\author{
Ching-Hua Kuo ${ }^{\mathrm{a}, \mathrm{b}, *}$, Chia-Wen Lee ${ }^{\mathrm{a}}$, Shu-Chiao Lin ${ }^{\mathrm{a}}$, I-Lin Tsai ${ }^{\mathrm{a}}$, Shoei-Sheng Lee ${ }^{\mathrm{a}, \mathrm{c}}$, \\ Y. Jane Tseng ${ }^{\mathrm{a}, \mathrm{d}}$, Jaw-Jou Kang ${ }^{\mathrm{c}, \mathrm{e}}$, Fu-Chuo Peng ${ }^{\mathrm{e}}$, Li Wei-Chu ${ }^{\mathrm{f}}$ \\ a School of Pharmacy, College of Medicine, National Taiwan University, Taipei, Taiwan \\ ${ }^{\mathrm{b}}$ Department of Pharmacy, National Taiwan University Hospital, Taipei, Taiwan \\ ${ }^{c}$ Drug Research Center, College of Medicine, National Taiwan University, Taipei, Taiwan \\ d Graduate Institute of Biomedical Electronic and Bioinformatics, National Taiwan University, Taipei, Taiwan \\ e Institute of Toxicology, College of Medicine, National Taiwan University, Taipei, Taiwan \\ ${ }^{f}$ Sheng Chang Pharmaceutical Co., Ltd., Jung-Li, Taiwan
}

\section{A R T I C L E I N F O}

\section{Article history:}

Received 19 June 2009

Received in revised form 2 October 2009

Accepted 5 October 2009

Available online 12 October 2009

\section{Keywords:}

Aristolochic acid

Herbal products

Aristolochic acid nephropathy

UHPLC-MS/MS

Biological samples

\begin{abstract}
A B S T R A C T
Aristolochic acids (AAs) are a mixture of structural-related compounds, in which aristolochic acid I (AA I) and aristolochic acid II (AA II) are reported to be correlated with Aristolochic acid nephropathy (AAN). In this work, a rapid and sensitive ultra-high-pressure liquid chromatography-tandem mass spectrometry (UHPLC-MS/MS) method was developed to determine AA I and AA II in herbal products and biological fluids. By using gradient elution with a mobile phase composed of a mixture of $10 \mathrm{mM}$ ammonium formate buffer ( $\mathrm{pH}$ 3.0) and acetonitrile, AAs could be determined within $10 \mathrm{~min}$. Under optimum UHPLC-MS/MS conditions, the limit of detections was 0.14 and $0.26 \mathrm{ng} \mathrm{mL}^{-1}$ for AA I and AA II, respectively. Run-to-run repeatability and intermediate precision of peak area for AA I and AA II were less than $5.74 \%$ relative standard deviation (RSD). Accuracy was tested by spiking 10,100 and $1000 \mathrm{ng} \mathrm{mL}^{-1}$ in rat serum and the recoveries were within 76.5-92.9\%. Matrix effects were within 78.8-127.7\%. The developed method was successfully applied to determine AA I and AA II in several herbal products and to investigate their pharmacokinetic behavior in female Wister rats. The result shows that the developed UHPLC-MS/MS method is efficient, sensitive, and accurate for the determination of AA I and AA II in herbal products and biological samples.
\end{abstract}

(c) 2009 Elsevier B.V. All rights reserved.

\section{Introduction}

Aristolochic acids (AAs) are found primarily in the plant genera Aristolochia and Asarum [1]. Pharmacological properties of AAs are anti-inflammatory, antitussive, antiplatelet aggregation. And they are also used as pain-relievers [2-4]. Inadvertent replacement of the plant Stephania tetrandra with Aristolochia fangchi in Chinese herbal medicines has caused many renal fibrosis problems, named Aristolochic acid nephropathy (AAN), in young women on a sliming regimen [5]. AAs were proved to be the major components that caused such toxicity [6]. Due to the serious side effect, USA, UK, Canada, Australia, and Germany government agencies

* Corresponding author at: School of Pharmacy, College of Medicine, National Taiwan University, 1, Section 1, Jen-Ai Road, Taipei 100, Taiwan. Tel.: +886 2 23123456x88394; fax: +886223919098.

E-mail address: kuoch@ntu.edu.tw (C.-H. Kuo). have banned the use of a range of species of plants that contain AAs. For similar reasons, several Chinese herbal medicines, including Aristolochia fangchi (Guangfangji), A. debilis (Qingmuxiang), A. manshuriensis (Guanmuton), and A. contorta (Madouling), have been prohibited to be imported or manufactured in Taiwan since 2003.

Despite the warning and import alert issued by the US Food and Drug Administration that herbal products containing AAs are unsafe [7], 19 products containing AAs and 95 products suspected to contain AAs were found to be sold on the Web in $2003[8,9]$. A recent study used capillary electrophoresis with laser-induced fluorescence to analyze 61 herbal products, and found that 24 samples suspected to contain AAs [10]. One way to reduce the incidence is by controlling the quality of herbal preparation via regular checking. Therefore, it is essential to develop sensitive and efficient analytical methods for the determination of AAs.

AAs are a mixture of structural-related 10-nitrophenanthrene 1-carboxylic acids [11], where AA I, the 8-methoxy-3,4- 
methylenedioxy derivative, and AA II, the 3,4-methylenedioxy derivative, are the major components in the Aristolochia species [12]. Thin layer chromatography [13], high performance liquid chromatography (HPLC) with UV absorption detection [14-16] and capillary electrophoresis (CE) with UV detection $[17,18]$ had been developed to analyze AA I and AA II in herbal products. Recently, HPLC coupled with fluorescence detection [19-21], CE coupled with laser-induced fluorescence detection [10], electrochemical detection [22], and on-line concentration technique [23] were developed to improve the sensitivity in detection.

HPLC and mass spectrometry hyphenated technique (HPLC-MS) has been proven to be a powerful tool in determining AAs in herbal products [24-29]. Due to the complexity in sample constituents, matrix effect is found to be remarkably significant in herbal extracts. The importance of eliminating matrix effect and ion suppressions during the development of quantitative methods based on HPLC-MS has been demonstrated in [30]. Various approaches have been used to eliminate matrix effect, including: (1) changing and improving sample extraction procedure by eliminating undetected matrix interferences, (2) performing the assay under more efficient chromatographic conditions to separate analytes of interest from other components, and (3) evaluating and changing the HPLC-MS interface [31]. The first two approaches, however, dramatically increase the analytical time, while the third approach may sacrifice sensitivity. In general, using the HPLC-MS/MS method to determine AAs in herbal products normally takes 20-60 min for an analysis [24-29].

Ultra-high-pressure liquid chromatography (UHPLC) is a recently developed technique which shows great advantages in providing better efficiency, sensitivity, and speed over ordinary HPLC. UHPLC columns packed with sub-2- $\mu \mathrm{m}$ particles can significantly increase the theoretical plate. Also, the increase in flow rate does not have negative impact on efficiency [32-34]. With sharper peaks and improved chromatographic resolution, matrix effect could be minimized without increasing the separation time [35]. There was one work which uses UHPLC-MS to analyze AAs, in which UHPLC-MS was applied to fingerprint analysis of AAs-containing preparations [36]. It used principal components analysis (PCA) and partial least square-discriminate analysis (PLS-DA) to identify the existence of AAs in pharmaceutical preparations. Due to the lack of selectivity and sensitivity consideration in that work, it is impractical to use the proposed method to quantitatively determine AAs in herbal preparations and biological samples. And the applicability to analyze biological samples is essential for the evaluation of nephrotoxicity. Up to now, there have been only two studies which investigated pharmacokinetic properties of AAI and AAII in vivo. Both of them used rabbits as their study animals because their analytical methods require a large sample volume $[37,38]$. The restriction of applying those methods to analyze rat serum limits the applicability of their approaches. Several studies revealed that AAs nephrotoxicity could be regulated by enzyme activity $[39,40]$. Since the pharmacokinetic properties of AAs are still unclear, it is necessary to develop a sensitive analytical method to facilitate these studies.

The primary goal of our research is to develop a sensitive and efficient UHPLC-MS/MS method to satisfy the demands on the aspects described earlier. To reach this goal, UHPLC-MS/MS conditions were systemically optimized for fast separation and sensitive detection. We used various herbal products, plasma samples, microsome incubation samples to demonstrate the practicability of the developed method. To the best of our knowledge, this is the first study using UHPLC-MS/MS to quantitatively analyze AA I and AA II in herbal products and biological samples.

\section{Experimental}

\subsection{Chemicals}

An AAs standard containing $90.9 \%$ of AA I and $5.7 \%$ of AA II was obtained from Acros (Geel, Belgium). Ammonium formate and ammonium acetate were obtained from Fluka Chemicals (Buchs, Switzerland). Methanol (MeOH) and acetonitrile (ACN) of chromatography grade were obtained from Mallinckrodt (Phillipsburg, NJ, USA). MeOH, ACN, and water of LC-MS grade were obtained from J.T. baker (Philipsburg, NJ). Dried fruits of $A$. contorta was collection from Liaoning, China in 2007. Dried roots of $A$. debilis was collected from Guangdong, China in 2007 and dried roots of $A$. fangchi were collected from Guangdong, China in 2007. The herbal samples were authenticated by Dr. Wei-Chu Li (Sheng Chang Pharmaceutical Corporation, Jhongli City, Taoyuan Country, Taiwan). The identification lab has laboratory accreditation issued by Taiwan accreditation foundation. Voucher specimens were stored at the Sheng Chang Pharmaceutical Corporation Herbarium. The concentrated powders of the decoction Bu-fei-a-jiau-tang was provided by Sheng Chang pharmaceutical co. (Chung-Li, Taiwan) under a research project contract (CCMP96-RD-044). Hsiao-Ching-LungTang, Chuan-Chiong-Char-Tiao-San and Shin-Yi-San were obtained in the Taipei market (Taipei, Taiwan).

All solutions were filtered through 0.2- $\mu \mathrm{m}$ GH Polypro filters before use. All samples were filtered through 0.22- $\mu \mathrm{m}$ PVDF filters before use (Millipore, Bedford, MA, USA).

\subsection{Isolation of $A A$ I and $A A$ II from AAs standard mixture}

AA I and AA II standards were separated by semi-preparative RP-HPLC. The mobile phase was an ammonium acetate buffer-ACN mixture $(72: 28, v / v)$. The buffer was prepared by adjusting $10 \mathrm{mM}$ ammonium acetate to $\mathrm{pH} 3.0$ with acetic acid. The flow rate was $2 \mathrm{~mL} \mathrm{~min}^{-1}$, and the detection wavelength was $254 \mathrm{~nm}$. The AAs standard mixture was dissolved in tetrahydrofuran at a concentration of $10 \mathrm{mg} \mathrm{mL}^{-1}$. The injection amount was $100 \mu \mathrm{L}$.

The equipments consisted of a Hitachi UV Pump L-7100 (Kyoto, Japan), a Rheodyne 7725i 5-mL manual injector (Cotati, CA, USA) and a Hitachi L-7400 UV detector. Separations were carried out on a Hibar Purospher RP-18 column (250 mm × 10 mm, $5 \mu \mathrm{m}$ ) (Merck, Darmstadt, Germany).

Stock solution ( $\left.1000 \mu \mathrm{g} \mathrm{mL} \mathrm{m}^{-1}\right)$ was prepared by dissolving $1 \mathrm{mg}$ AA I and $1 \mathrm{mg} \mathrm{AA} \mathrm{II} \mathrm{in} 1 \mathrm{~mL}$ MeOH separately. Adequate amounts of the stock solution were diluted with $\mathrm{MeOH}$ to prepare for the standard solutions for the respective experiment.

\subsection{Apparatus}

\subsubsection{UHPLC-DAD}

The equipments consisted of a Waters Acquity UPLC autosampler, column manager and thermostat, binary solvent manager, and an Acquity UPLC diode array detector (Waters, Milford, MA). The UV detection range was $200-400 \mathrm{~nm}$. Separations were carried out on a Waters Acquity UPLC BEH C18 column $(100 \mathrm{~mm} \times 2.1 \mathrm{~mm}, 1.7 \mu \mathrm{m})$.

\subsubsection{UHPLC-MS/MS}

The Acquity UPLC system consisted of an autosampler, column manager with thermostat, a degasser, and a binary solvent manager (Waters, Milford, MA).

A Quatro Micro triple-quadrupole mass spectrometer (Micromass, Manchester, UK) equipped with an electrospray ionization (ESI) source was used. The mass spectrometer was operated in the positive ion electrospray mode with selective reaction monitoring (SRM). The analyses were performed in positive mode with a cone voltage of $20 \mathrm{~V}$ for AA I and $20 \mathrm{~V}$ for AA II, extractor voltage of $3.0 \mathrm{~V}$, 
$\mathrm{RF}$ lens voltage of $0.0 \mathrm{~V}$, capillary voltage of $3.0 \mathrm{kV}$, ion source temperature of $80^{\circ} \mathrm{C}$, and desolvation temperature of $450^{\circ} \mathrm{C}$. A cone gas flow of $50 \mathrm{Lh}^{-1}$ and desolvation gas flow of $1100 \mathrm{Lh}^{-1}$ were used. Argon (99.999\%) was used as a collision gas. Gas flow rate was kept at $0.13 \mathrm{~nL} \mathrm{~min}{ }^{-1}$ in the collision cell. The collision energy was $12 \mathrm{eV}$ for AA I and $10 \mathrm{eV}$ for AA II. MassLynx 4.1 software was used for data analysis.

Chromatographic separation was performed by using an Acquity BEH C18 column $(100 \mathrm{~mm} \times 2.1 \mathrm{~mm}, 1.7 \mu \mathrm{m})$ (Waters, Milford, MA). The mobile phase was composed of $10 \mathrm{mM}$ ammonium formate ( $\mathrm{pH}$ 3.0) (solvent $\mathrm{A}$ ) and ACN (solvent $\mathrm{B}$ ). The gradient profile was: $0-2$ min: $34 \% \mathrm{~B}$; $2-10.5 \mathrm{~min}$ : linear from $34 \%$ to $36 \% \mathrm{~B}$; 10.5-10.6 min: linear from $36 \%$ to $34 \% \mathrm{~B}$ and then re-equilibrium of the column. The flow rate was kept at $0.3 \mathrm{~mL} \mathrm{~min}^{-1}$ The column oven was set at $40^{\circ} \mathrm{C}$ and the auto-injection system was set at $4{ }^{\circ} \mathrm{C}$. Partial loop with needle overfill mode was used for sample injection, and the injection volume was $5 \mu \mathrm{L}$.

\subsection{Pharmacokinetic study of AA I in rat}

\subsubsection{Drug administration and sample collection}

AAs standard powder ( $90.9 \%$ of AA I and $5.7 \%$ of AA II) was dissolved in corn oil at a concentration of $0.5 \mathrm{mg} \mathrm{mL}^{-1}$. The AA standard solution was administered to female Wistar rats at a dose of $10 \mathrm{mg} \mathrm{kg}^{-1}$ through an intragastric tube. Blood samples $(0.2 \mathrm{~mL})$ were taken from the femoral vein at $15,30,45,60,90,120,240$, $360,480,600 \mathrm{~min}$ after oral treatment. The blood samples were centrifuged and the serum was taken and stored at $-80^{\circ} \mathrm{C}$ until analyzed by the UHPLC-MS/MS method.

\subsubsection{Preparation of liver microsomes}

Two adult male ICR mice were injected with $\beta$-naphthoflavone at a dose of $50 \mathrm{mg} \mathrm{kg}^{-1}$ for three consecutive days to induce liver CYP1A enzyme activities. Mice were sacrificed by cervical dislocation, and their livers were removed immediately on ice for further microsomes preparation.

Livers were homogenized in $1.15 \% \mathrm{KCl}$ buffer, and the mixture was centrifuged at $8000 \mathrm{rpm}$ for $20 \mathrm{~min}$ at $4{ }^{\circ} \mathrm{C}$. The supernatant was further subjected to ultracentrifuge at 39,000 rpm (Beckman, Fullerton, CA) for $60 \mathrm{~min}$. The pellet was rinsed with $1.15 \% \mathrm{KCl}$ buffer and then ultracentrifuged again. The pellet was collected and dissolved in equal amount of $100 \mathrm{mM} \mathrm{K}_{2} \mathrm{HPO}_{4}(\mathrm{pH} 7.4)$.

The microsomal protein concentration was determined by BCA protein assay (Thermo Fisher Scientific, Rockford, IL).

The animal study presented in this paper was approved by National Taiwan University, College of Medicine, and College of Public Health Institutional Animal Care and Use Committee (IACUC Approval No: 20070151).

\subsection{Sample preparation}

\subsubsection{Extraction of plant material}

Aristolochia plants and their pharmaceutical preparations were grounded to powder. Two grams of each powder were ultrasonically extracted with $10 \mathrm{~mL}$ of $\mathrm{MeOH}$ for $10 \mathrm{~min}$ and followed by centrifugation at $8000 \mathrm{rpm}$ for $5 \mathrm{~min}$. The extraction was repeated for three times and the supernantants were combined and concentrated under reduced pressure until dry. Suitable amount of the residue was re-dissolved with $\mathrm{MeOH}$ for the respective experiment.

\subsubsection{Serum sample pretreatment}

One hundred and sixty microliters of ACN was added to $80 \mu \mathrm{L}$ serum for deproteination. After $15 \mathrm{~min}$, the mixture was centrifuged at $12,000 \mathrm{rpm}$ for another $15 \mathrm{~min}$ to remove proteins. Two hundred microliter of supernatant was evaporated to dryness under nitrogen flow, and then reconstituted in
$\mathrm{MeOH}$. The reconstituted solution was subjected to UHPLC-MS/MS analysis.

\subsubsection{Microsome incubation sample}

The incubation mixture (final volume $0.75 \mathrm{~mL}$ ) contained $0.75 \mathrm{mg}$ mice liver microsomes, $0.19 \mathrm{mM} \mathrm{NADPH}, 1.56 \mathrm{mM} \mathrm{MgCl}_{2}$ $0.1 \mathrm{M}$ sodium phosphate buffer ( $\mathrm{pH} 7.4$ ) and $0.3 \mathrm{mM} \mathrm{AAI}$ standard. The $0.3 \mathrm{mM}$ of AAI standard was replaced with Bu-fei-a-jiau-tang in the study of herbals mixture study. The mixture was shaken (100 oscillations/min) for $5 \mathrm{~min}$ at $37^{\circ} \mathrm{C}$. Then the substrate was added to trigger the reaction. After $30 \mathrm{~min}$ of incubation with shaking at $37^{\circ} \mathrm{C}$, the reaction was stopped by adding $0.2 \mathrm{~mL}$ of methanol and the mixture was centrifuged at $39,000 \mathrm{rpm}$ for $25 \mathrm{~min}$ at $4{ }^{\circ} \mathrm{C}$. Part of the supernatant $(100 \mu \mathrm{L})$ was mixed with $100 \mu \mathrm{L}$ ACN After $15 \mathrm{~min}$, the mixture was centrifuged at $3000 \mathrm{rpm}$ for $5 \mathrm{~min}$ to separate precipitated proteins. The supernatant was then transferred into a $1.5-\mathrm{mL}$ tube containing $50-60 \mathrm{mg}$ sodium chloride. The suspension was vortex-mixed briefly and incubated at room temperature for $20 \mathrm{~min}$ and vortex-mixing again. Finally, the mixture was centrifuged at $3000 \mathrm{rpm}$ for $5 \mathrm{~min}$, and then was subjected to UHPLC-MS/MS analysis.

\section{Results and discussion}

Parameters affecting UHPLC-MS/MS performance were systemically optimized in this study.

\subsection{Optimization of UHPLC-MS/MS conditions}

AA I and AA II tends to form ammonia adduct in the mobile phase [24]. The precursor ion were set at $m / z 359\left[\mathrm{AA} \mathrm{I}+\mathrm{NH}_{4}\right]^{+}$ and $m / z 329$ [AA II $\left.+\mathrm{NH}_{4}\right]^{+}$to provide the best detection sensitivity. The most intense precursor-product transitions were selected as the quantification transitions in the multiple reaction monitoring (MRM) mode. The fragmentation mass spectra of the ion $\left[\mathrm{M}+\mathrm{NH}_{4}\right]^{+}$ derived from AA I and AA II are shown in Fig. 1. As depicted in the figures $\left[\mathrm{M}+\mathrm{H}-\mathrm{CO}_{2}\right]^{+}$was the base peak for both analytes. The fragment ions at $m / z 298\left[\mathrm{AA} \mathrm{I}+\mathrm{H}-\mathrm{CO}_{2}\right]^{+}$and $m / z 268\left[\mathrm{AA} \mathrm{II}+\mathrm{H}-\mathrm{CO}_{2}\right]^{+}$ were therefore selected as the product ions. Both APCI (+) and ESI $(+)(-)$ modes have been reported as the LC-MS ionization source in AAs analysis [21-26]. APCI with a mobile phase flow rate at $0.5 \mathrm{~mL} \mathrm{~min}^{-1}$ and ESI with a mobile phase flow rate at $0.3 \mathrm{~mL} \mathrm{~min}{ }^{-1}$ were tested for their ionization efficiency. Signal intensity obtained under ESI $(+)$ was found to be higher than that under APCI (+) over ten fold. Thus, ESI $(+)$ was used as the ionization source in our study.

The UHPLC mobile phase was optimized to provide the best selectivity and the highest signal intensity in this study. The structures of both AA I and AA II contain one carboxylic acid group with a $\mathrm{p} K_{\mathrm{a}}$ value being 3.2-3.4 and 3.3-3.5, respectively [41]. Due to the acidic properties of AA I and AA II, the acidic mobile phase gave better retention and chromatographic separation in the reversed phase column. The addition of buffer also assisted the ESI analysis. The influence of the buffer type and the $\mathrm{pH}$ value of the mobile phase on selectivity and sensitivity were further investigated.

\subsubsection{Optimization of UHPLC mobile phase}

Kite et al. had studied the effect of the LC buffer type on MS signal intensities of AAs [24]. In their study, $0.1 \%$ ammonium acetate gave the best results. The buffer type investigation presented by Chan showed that the addition of $1.0 \mathrm{mM}$ ammonium acetate in $0.2 \%$ acetic acid dramatically increases the signal of the ammonium adduct [26]. In this study, mobile phase with the use of $10 \mathrm{mM}$ ammonium acetate and $10 \mathrm{mM}$ ammonium formate as buffer solution were compared to see their effect on MS peak intensity. The intensities of MS signals were shown in Fig. 2. It was found that peak intensity obtained under ammonium formate system was more 

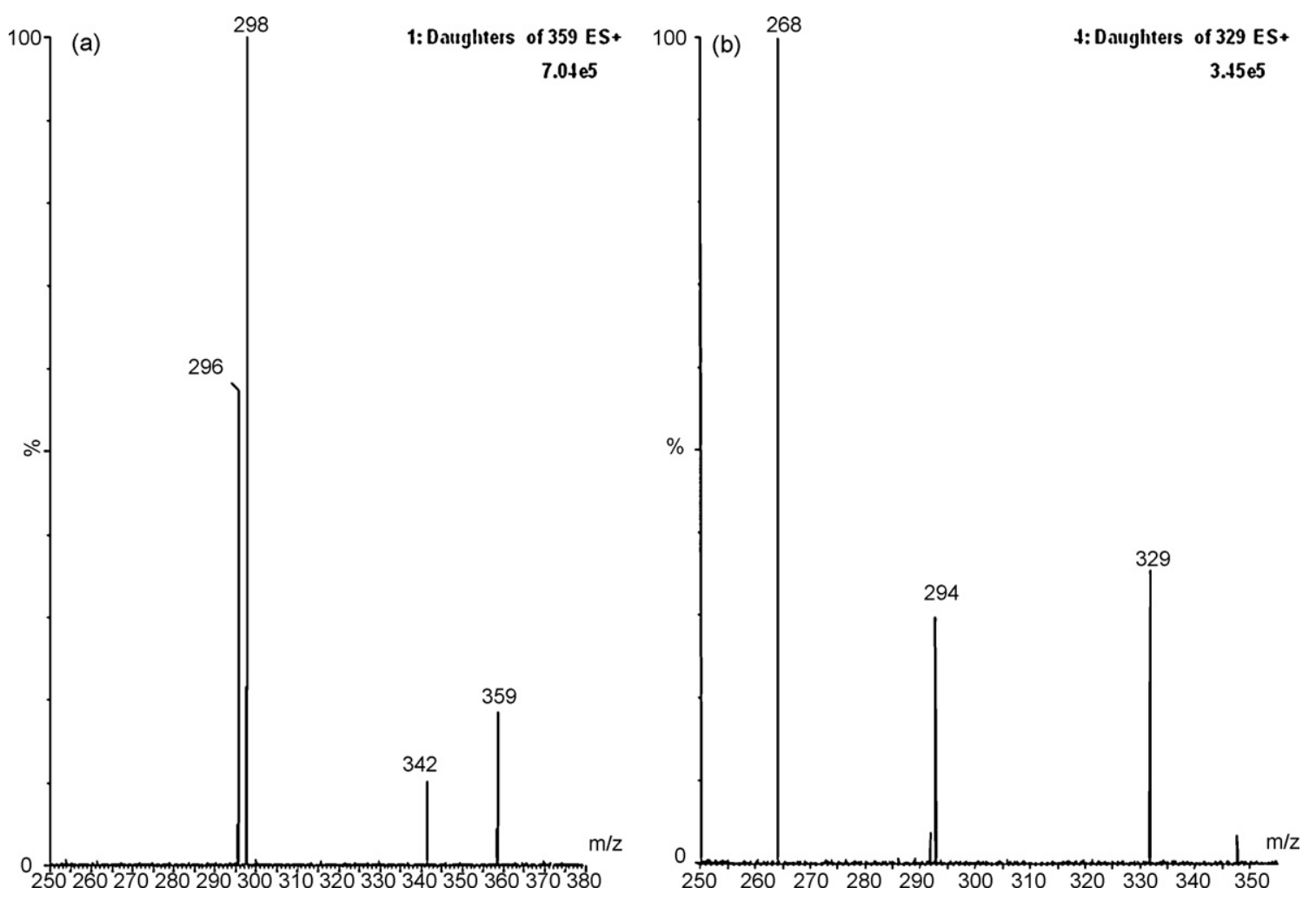

Fig. 1. MS/MS spectra of AA I (a) and AA II (b).

than two fold higher than that under ammonium acetate system. Therefore, $10 \mathrm{mM}$ ammonium formate was selected to be the buffer system. The influence of organic solvent on the peak intensity was also investigated. Peak efficiencies were better when ACN instead of $\mathrm{MeOH}$ was used as the organic solvent in the mobile phase. Moreover, signal intensities obtained under the ACN system was higher than those under the $\mathrm{MeOH}$ system for more than five fold. ACN was therefore chosen as the organic solvent in the mobile phase.

Because radioactive isotope-labeled internal standards of AA I and AA II were not commercially available, the higher resolution provided by UHPLC played an important role in reducing the matrix effect. This is especially important for complicated samples such as herbal products. The $\mathrm{p} K_{\mathrm{a}}$ value of AA I is between 3.2 and 3.4, and that of AA II is between 3.3 and 3.5. It was expected that in the mobile phase, various $\mathrm{pH}$ values from 3 to 5 would show significant effect on their retention. Several herbals containing AAs were

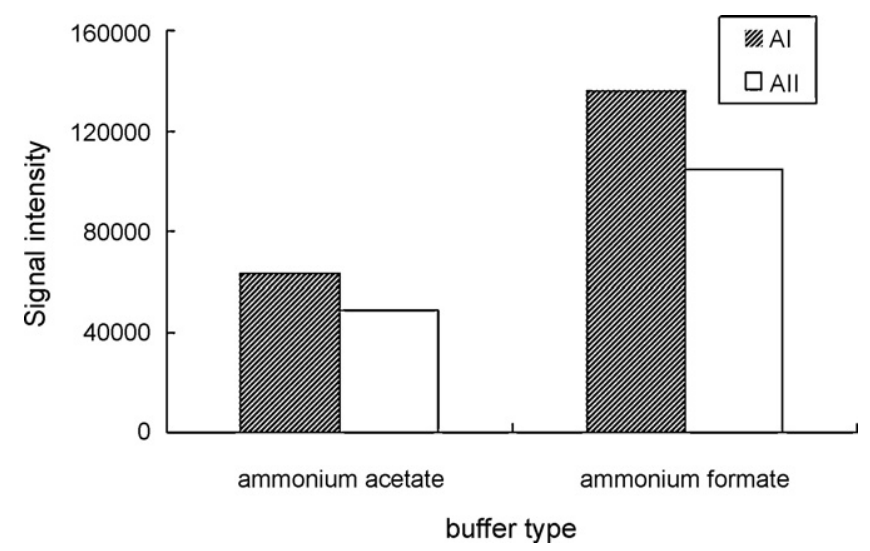

Fig. 2. Effect of buffer type on peak intensity of AAI and AAII in UHPLC-MS/MS system. tested in this experiment to evaluate the resolution of AA I and AA II from the complicated plant constituents. In order to test the selectivity of the developed conditions to herbals mixture, we used Bu-fei-a-jiau-tang as the tested formula. It was observed that the retention time of $A A$ I and $A A$ II increased while the $\mathrm{pH}$ value was decreased because AAs tend to be in their free form in more acidic conditions. The best resolution was obtained at $\mathrm{pH}$ 3.0. Gradient shape, gradient steepness and gradient range of the mobile phase were subsequently studied for the optimum separation conditions. The optimum gradient profile was: 0-2 min: 34\% ACN; 2-10.5 min: linear from $34 \%$ to $36 \% \mathrm{ACN}$ and then re-equilibrium of the column. UHPLC-UV and UHPLC-MS/MS chromatograms of the herbal extracts obtained under the optimum separation conditions are shown in Fig. 3. The results show that AA I and AA II could be baseline-separated from other herbal constituents within $10 \mathrm{~min}$ under optimum UHPLC conditions.

\subsubsection{Optimization of ESI interface and mass spectrometry conditions}

In the ESI mode, the distance from the emitter to the inlet has significant effect on mass sensitivity; at shorter distances, more of the ES plume will be sampled into the instrument. However, droplet evaporation time also reduces at smaller distances, resulting in lower ionization efficiency [42]. In this study, we found that the signals of AA I and AA II were intensified when the distance was increased from 3 to $4 \mathrm{~mm}$, but dramatically weakened when the distance was further increased up to $5 \mathrm{~mm}$, which was due to the low transmitted ES current. The value of $4 \mathrm{~mm}$ was consequently selected as the distance from the emitter to the inlet.

Cone voltage was shown to have the most profound effect on the signal intensity among all of the mass parameters. Signal intensity of AA I was increased more than four fold when the voltage was increased from 15 to $20 \mathrm{~V}$, but decreased significantly if the voltage was further increased. This was attributed to the disappearance of ammonia in the mixture at the high voltage. The phenomenon is 

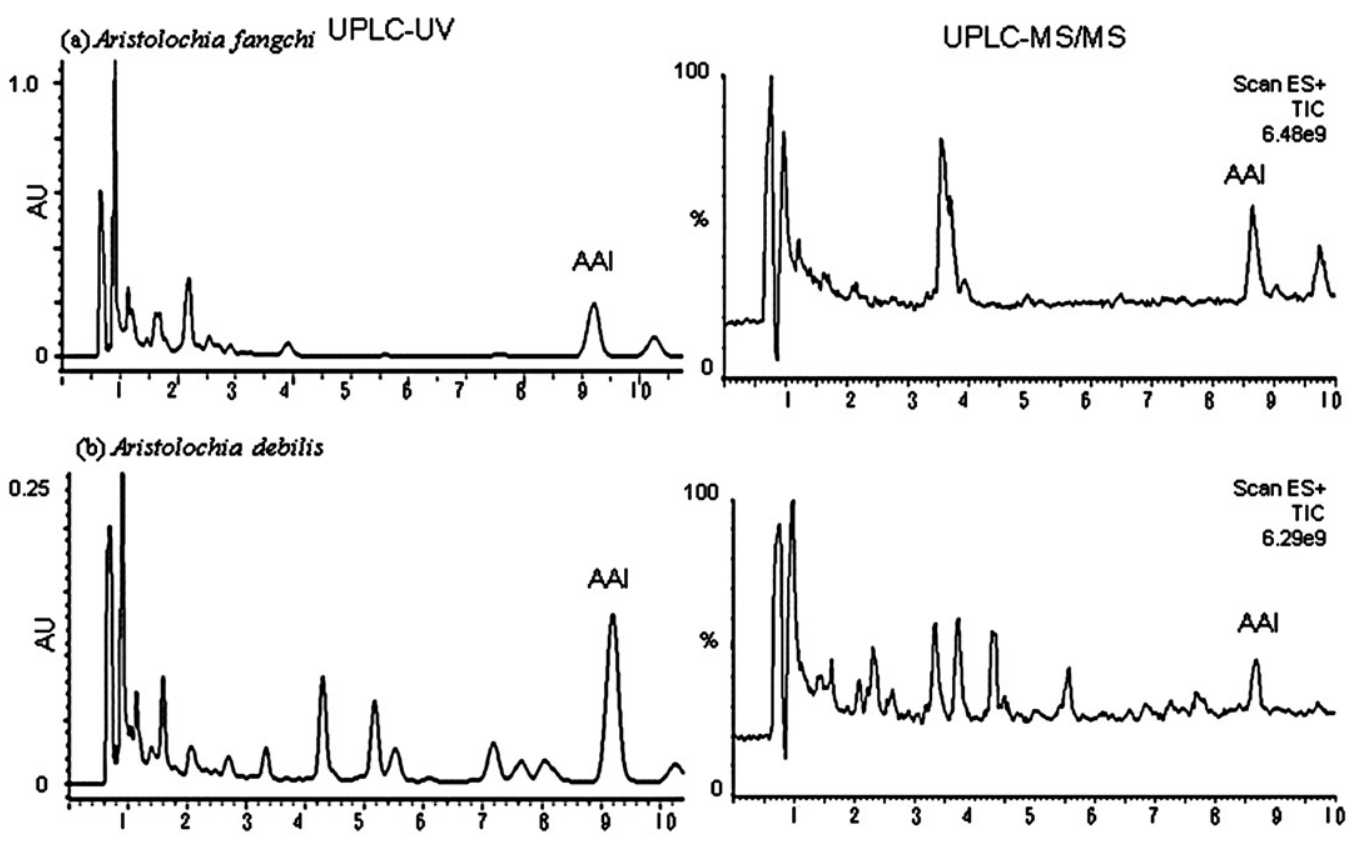

(c) Aristolochia contorta
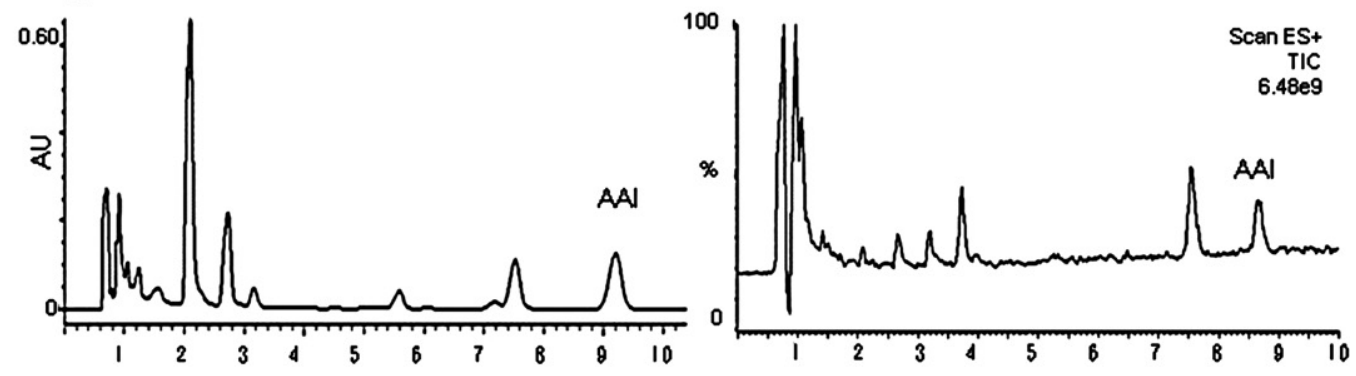

(d) Bu-fei-a-jiau-tang
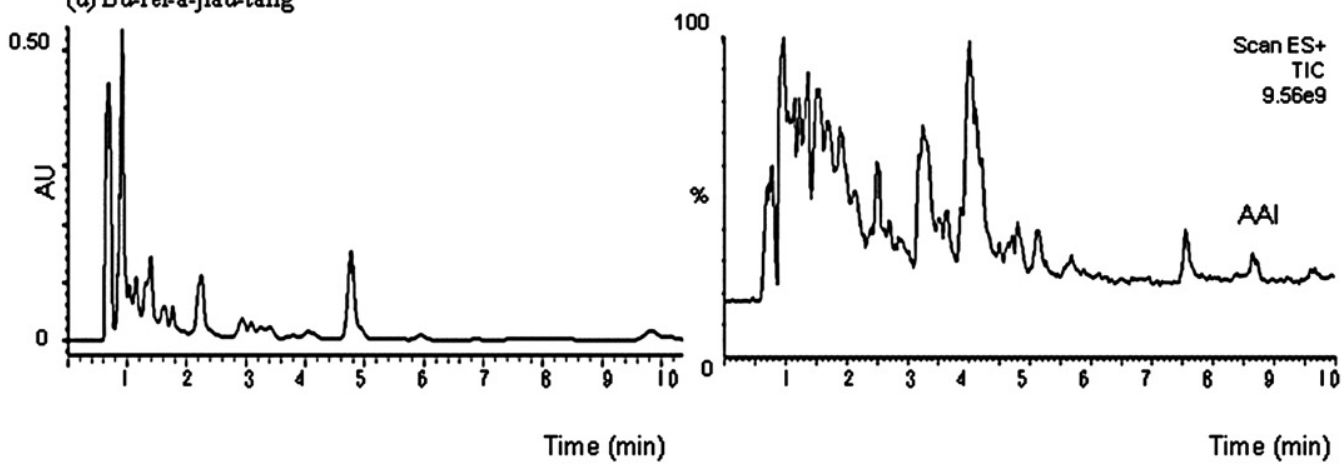

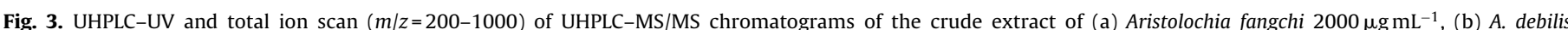

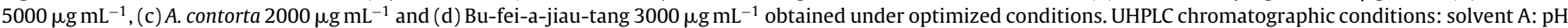

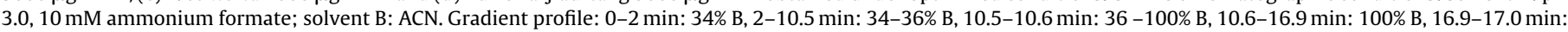

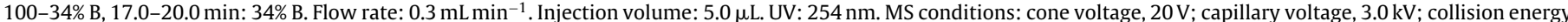

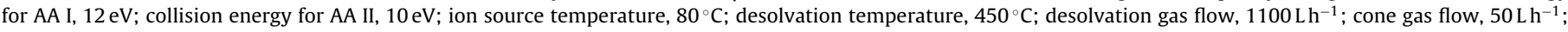
probe distance, $4 \mathrm{~mm}$.

consistent with what is reported by Kasai et al. [43]. Higher cone voltage should decrease the amount of ammonium-cation adduct. The value of $20 \mathrm{~V}$ was selected as the cone voltage for AA I and AA II analysis.

Since raising desolvation temperature would improve solvent evaporation and increase the amount of gas-phase ions and clusters, the desolvation temperature varied from 350 to $500^{\circ} \mathrm{C}$ was investigated. Signal intensities of AA I and AA II were found to be increasing with the rise of desolvation temperature up to the highest applicable $500^{\circ} \mathrm{C}$, which was therefore selected as the des- olvation temperature in this study. Collision energy larger than $20 \mathrm{eV}$ resulted in higher degree of fragmentation which led to lower signal intensity. Values of 12 and $10 \mathrm{eV}$ were selected for AA I and AA II, respectively. Other mass parameters (ion source voltage, temperature of the heated capillary, and flow rate of nitrogen gas) had minor influence on the mass sensitivity. Optimum values of these parameters are listed in the Section 2. Multiple reaction monitoring (MRM) chromatogram of AA I and AA II at $1 \mu \mathrm{g} \mathrm{mL}^{-1}$ obtained under the optimum conditions is shown in Fig. 4. 


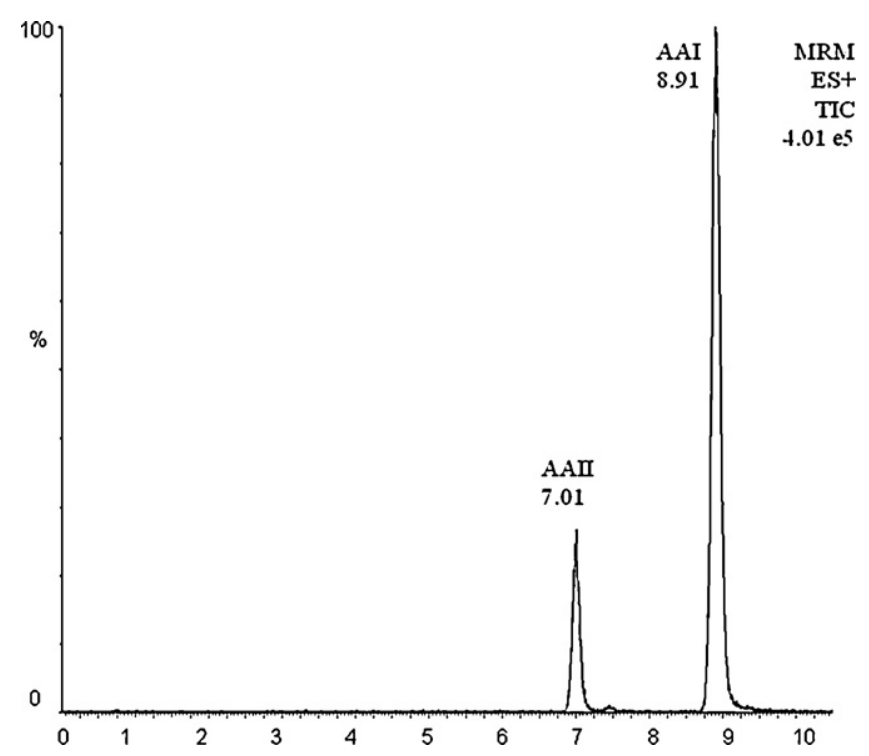

Fig. 4. UHPLC-MS/MS chromatogram (MRM) of AA I and AA II obtained under optimum conditions. UHPLC-MS/MS conditions are the same with those described in Fig. 3.

\subsection{Method validation}

\subsubsection{Precision and accuracy}

Run-to-run repeatability (intraday, $n=3$ ) and intermediate precision (interday, $n=3$ ) of migration time and peak area were tested. Both repeatability and intermediate precision of AA I and AA II in terms of migration time were within $1.25 \%$ relative standard deviation (RSD). The precision of peak areas of AA I and AA II were tested at 10,100 and $3000 \mathrm{ng} \mathrm{mL}^{-1}$. Repeatability $(n=3)$ and intermediate precision $(n=3)$ were within $3.99 \%$ and $5.74 \%$ RSD, respectively.

The accuracy of the method was studied by spiking AA I and AA II at 10,100 and $3000 \mathrm{ng} \mathrm{mL}^{-1}$ in blank rat serum. The recoveries were within $76.5 \pm 0.6 \%$ and $92.9 \pm 1.1 \%$, and the detailed data were shown in Table 1.

\subsubsection{Linearity}

Linearity of the method was tested with eight standard solutions of AA I and AA II at concentrations ranging from 10 to $3000 \mathrm{ng} \mathrm{mL}^{-1}$. Each solution was tested for three times. The regression equation is $y=84.501 x-99.882\left(r^{2}=0.9999\right)$ for AA I, and $y=36.363 x-207.87$ $\left(r^{2}=0.9999\right)$ for AA II.

\subsubsection{Limit of detection ( $L O D)$ and limit of quantification ( $L O Q)$}

LOD of the method was defined as the concentration of AA I and AA II detected at $\mathrm{S} / \mathrm{N}=3$. It was found to be $0.14 \mathrm{ng} \mathrm{mL}^{-1}$ for $\mathrm{AA} I$ and $0.26 \mathrm{ng} \mathrm{mL}^{-1}$ for AA II. LOQ of this method was determined with a concentration of $A A$ I and $A A$ II at $S / N=10$. Its value was found to be $0.5 \mathrm{ng} \mathrm{mL}^{-1}$ for AA I and $0.7 \mathrm{ng} \mathrm{mL}^{-1}$ for AA II.

\subsubsection{Matrix effect}

Matrix effects may cause ion suppression or ion enhancement. Their magnitude may vary between sample components. Standard

Table 1

Recovery of AAI and AAII in rat serum.

\begin{tabular}{rlc}
\hline Concentration $\left(\mathrm{ng} \mathrm{mL}^{-1}\right)$ & \multicolumn{2}{l}{ Recovery (\%) } \\
\cline { 2 - 3 } & AAI & AAII \\
\hline 10 & $76.5 \pm 0.6$ & $80.4 \pm 1.8$ \\
100 & $83.6 \pm 3.5$ & $80.4 \pm 3.4$ \\
\hline
\end{tabular}

Table 2

Matrix effects of AA I and AA II in Aristolochia debilis and rat serum.

\begin{tabular}{|c|c|c|c|c|}
\hline \multirow[t]{3}{*}{ Concentration $\left(\mathrm{ng} \mathrm{mL}^{-1}\right)$} & \multicolumn{4}{|c|}{ Matrix effect (\%) } \\
\hline & \multicolumn{2}{|c|}{ Aristolochia debilis } & \multicolumn{2}{|l|}{ Rat serum } \\
\hline & AA I & AA II & AA I & AA II \\
\hline 10 & $127.7 \pm 10.5$ & $120.0 \pm 7.7$ & $78.8 \pm 3.9$ & $83.5 \pm 4.1$ \\
\hline 100 & $104.3 \pm 5.9$ & $95.6 \pm 3.0$ & $87.0 \pm 2.2$ & $88.0 \pm 3.2$ \\
\hline 1000 & $93.8 \pm 1.3$ & $95.8 \pm 1.9$ & $115.6 \pm 0.7$ & $113.0 \pm 2.6$ \\
\hline
\end{tabular}

solutions of 10,100 and $1000 \mathrm{ng} \mathrm{mL}^{-1}$ of AA I and AA II were added to the sample solutions of $A$. debilis and rat serum. Area of postextraction spike was calculated against area of standard in order to study the matrix effect. The test results are shown in Table 2 . Matrix effects of AA I and AA II in A. debilis and rat serum were within $127.7 \pm 10.5 \%$ to $78.8 \pm 3.9 \%$.

\subsection{Application}

\subsubsection{Herbal medicines}

Herbal products were generally given as mixtures of herbs. Therefore, both plants of Aristolochia-genius and traditional Chinese medicines containing plants with AAs were included to investigate the applicability of our developed method to real samples. The UHPLC-MS/MS method validated above was applied to analyze the crude extract of $A$. contorta (dried fruits), $A$. debilis (dried roots), A. fangchi (dried roots), A. heterotropoides (dried roots), Bu-fei-a-jiau-tang, Hsiao-Ching-Lung-Tang, ChuanChiong-Char-Tiao-San and Shin-Yi-San. Bu-fei-a-jiau-tang is an antitussives preparation which has been banned in Taiwan since 2003, Hsiao-Ching-Lung-Tang and Shin-Yi-San are used to relieve cold symptoms, and Chuan-Chiong-Char-Tiao-San is used for migraine. And these preparations are still available in the market. Total ion scan and MRM chromatograms of the four of the herbal products were shown in Figs. 3 and 5, respectively, The constituents in Bu-fei-a-jiau-tang were the most complicated among them. The contents of AA I and AA II are shown in Table 3. AAI, the compound predominantly responsible for the nephropathy and urothelial cancer of AAN [39], is much higher than AAII in all tested herbal products. Among the tested herbal products, A. debilis contains the highest amount of AAs. With the high sensitivity of the developed method, several herbal products which are still available in the market were detected to contain small amount of AAI. It is worthwhile to investigate the safety of long-term exposure to these products.

\subsubsection{Pharmacokinetics of $A A$}

With the high sensitivity of the developed UHPLC-MS/MS method, the pharmacokinetic parameters of AA I and AA II in rats can be investigated. AAs standard containing $90.9 \%$ of AA I and $5.7 \%$ of AA II was dissolved in corn oil and intragastrically administered to a female Wister rat at a dose of $10 \mathrm{mg} \mathrm{kg}^{-1}$. Blood samples were taken from femoral vein at the designed time intervals. Serum samples were subjected to protein denaturation and then analyzed by UHPLC-MS/MS. The serum concentration-time curves of AA I and AA II after oral administration of AAs are shown in Fig. 6. Table 4 presents the pharmacokinetics parameters of AA I and AA II calculated by WinNolin. The data revealed that AAs could be quickly absorbed in gastro-intestinal tract, and their $T_{\max }$ was within $1 \mathrm{~h}$.

The role of biotransformation enzymes in the development of renal injury caused by AAs remains unclear. Xiao et al. had performed in vivo and in vitro studies to investigate the correlation between hepatic cytochrom P450 (P450) enzyme activity and AAI toxicity. The conclusion was that hepatic P450 1A1 and $1 \mathrm{~A} 2$ could detoxify AAI through the metabolism of AAI into less toxic metabolites aristolactam I (AL I) [39]. In this study, we tested 

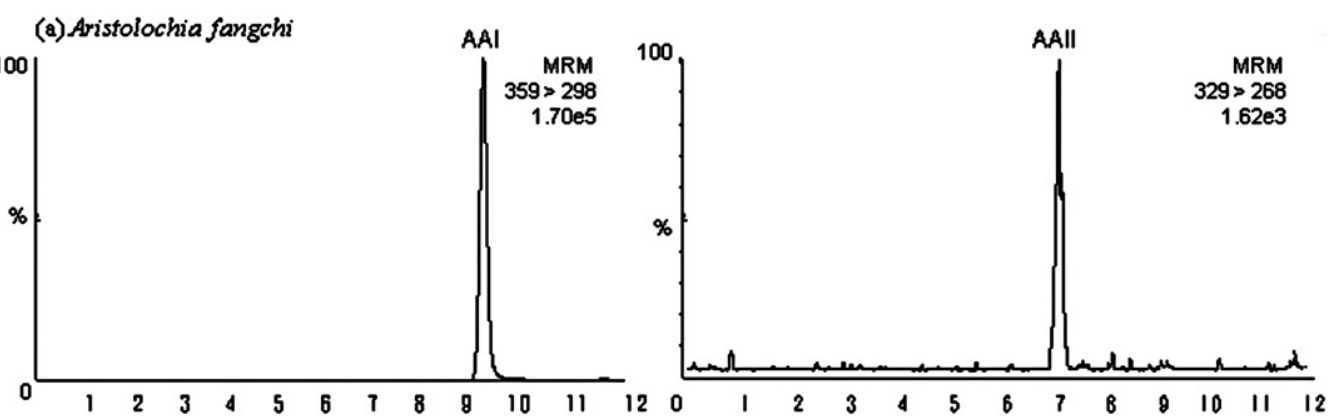

(b) Aristolochia debilis
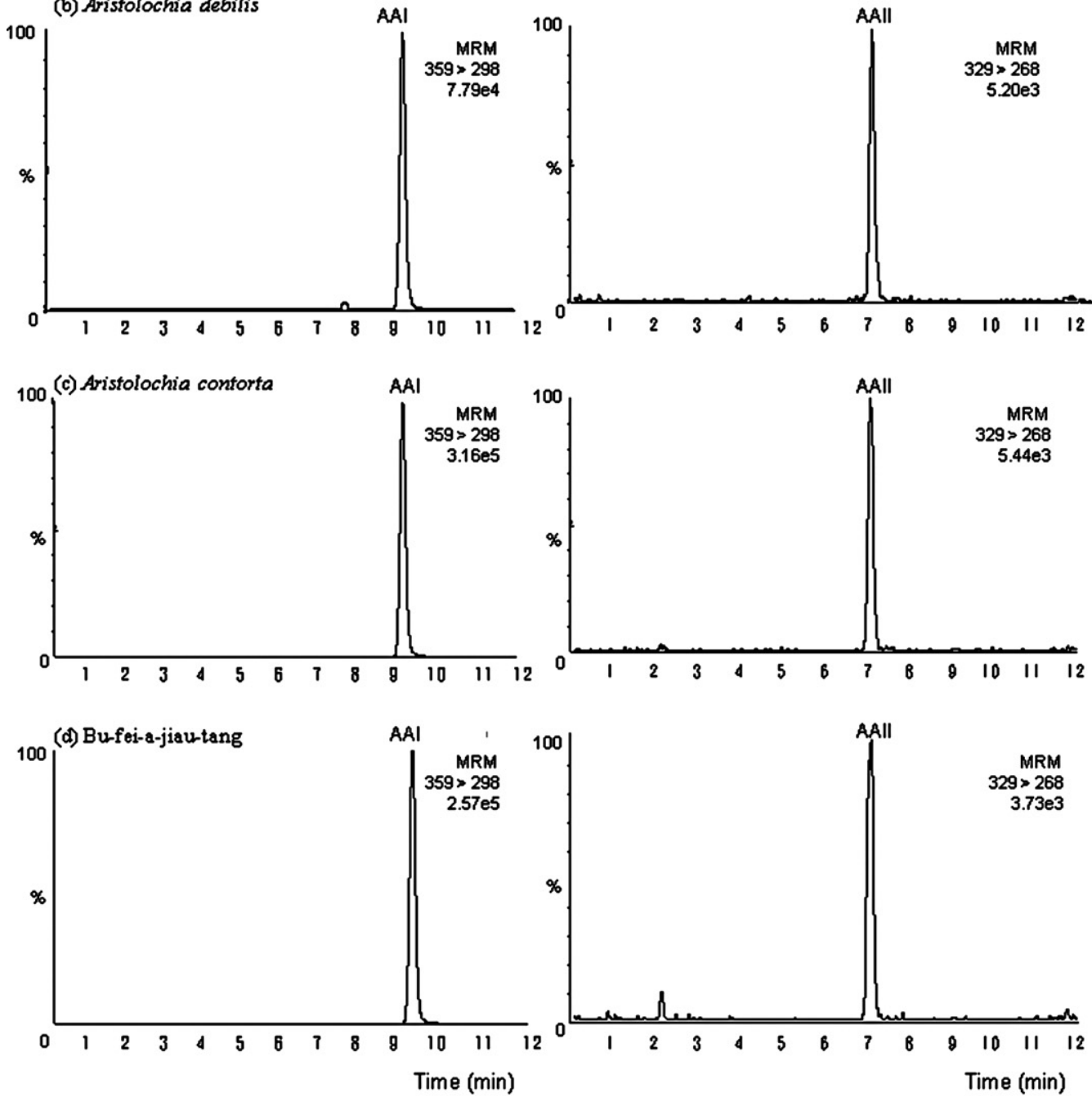

Fig. 5. MRM chromatograms of the crude extract of (a) Aristolochia fangchi, (b) A. debilis, (c) A. contorta and (d) Bu-fei-a-jiau-tang. UHPLC-MS/MS conditions are the same with those described in Fig. 3.

Table 3

Contents of AAI and AAII in herbal products.

\begin{tabular}{lcc}
\hline Content & AA I $\left(\mu \mathrm{gg}^{-1}\right)$ & \multicolumn{1}{c}{ AA II $\left(\mu \mathrm{gg}^{-1}\right)$} \\
\hline Aristolochia fangchi (fruits) & $944.569 \pm 36.206$ & $50.078 \pm 1.845$ \\
Aristolochia debilis (roots) & $802.565 \pm 38.238$ & $148.687 \pm 8.038$ \\
Aristolochia contorta (roots) & $511.478 \pm 19.275$ & $29.386 \pm 1.143$ \\
Asarum heterotropoides (roots) & $7.572 \pm 0.043$ & - $^{\mathrm{a}}$ \\
Bu-fei-a-jiau-tang & $119.674 \pm 3.298$ & $6.802 \pm 0.289$ \\
Hsiao-Ching-Lung-Teng & $0.194 \pm 0.001$ & - \\
Chuan-Chiong-Char-Tiao-San & $0.384 \pm 0.012$ & - \\
Shin-Yi-San & $0.525 \pm 0.001$ & - \\
\hline
\end{tabular}

a Below detection limit. the applicability of our developed method in the investigation of AAI metabolism. Both AA I standard and Bu-fei-a-jiau-tang were incubated with liver microsome. The microsome incubated samples were subjected to protein denaturation and then analyzed by UHPLC-MS/MS. The MRM chromatograms were shown in Fig. 7. AL I only appeared in sample treated with Bu-fei-a-jiau-tang. Since toxicity between AA I and AL I, are different, our finding showed that the toxicity of AA I standard and herbal mixtures containing AA I might be different. Although more experiments should be preformed to explain this phenomenon, our study showed that the developed UHPLC-MS/MS is a powerful tool to investigate the pharmacokinetic properties of AAs. 

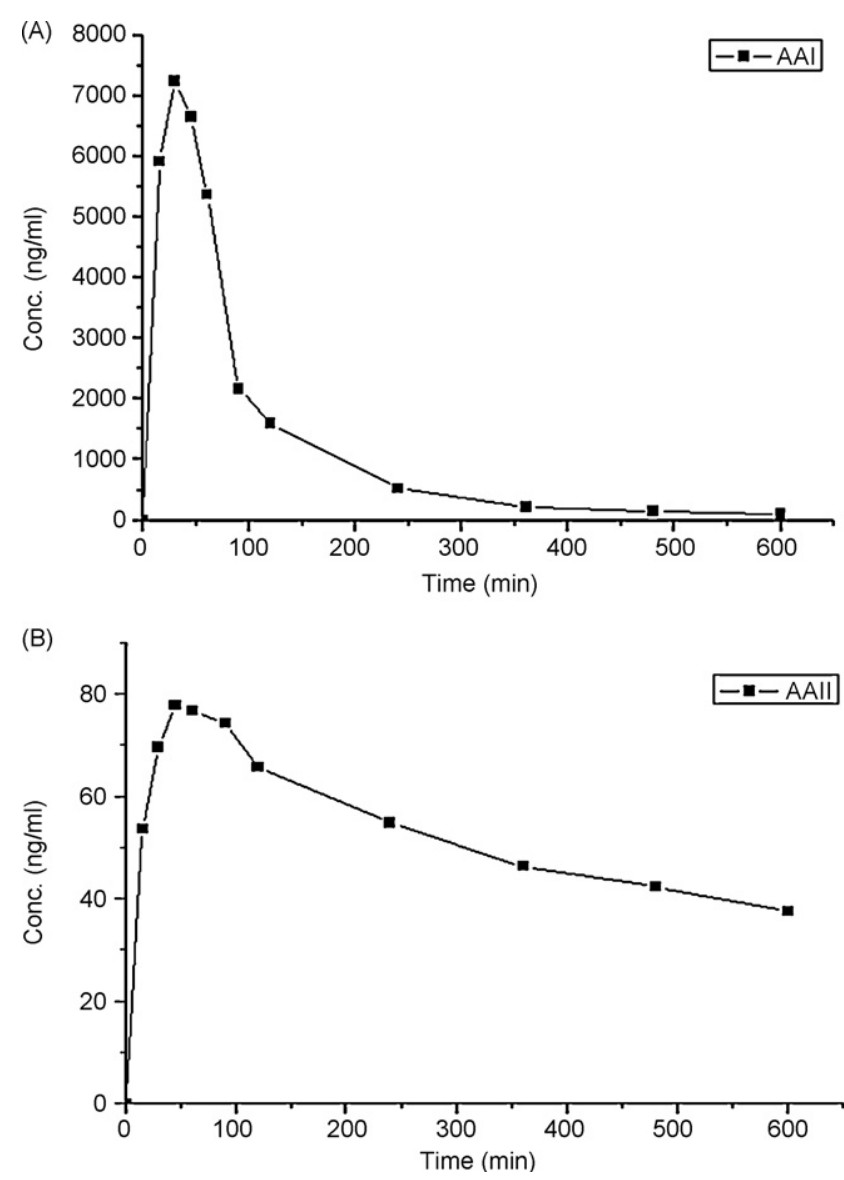

Fig. 6. Serum concentration-time plots of AA I (A) and AA II (B) after oral administration of $A A s$ at a dose of $10 \mathrm{mg} \mathrm{kg}^{-1}$ in female Wister rats.

\subsection{Performance of UHPLC-MS/MS}

Several HPLC-UV methods have been applied to analyze AAs in plants similar to this study $[14-16,19,25]$. The analytical time of those studies were all longer than $15 \mathrm{~min}$. Other studies used the HPLC-MS methods and their analytical time were higher than $12 \mathrm{~min}$. Also the LODs of these methods were higher than $3.99 \mathrm{ng} \mathrm{mL}^{-1}$ for AAI and $3.71 \mathrm{ng} \mathrm{mL}^{-1}$ for AAII $[26,28,29]$. By using the presented UHPLC-MS/MS method, AAI and AAII could be determined within $10 \mathrm{~min}$, and the LODs were $0.14 \mathrm{ng} \mathrm{mL}^{-1}$ for AA I and $0.26 \mathrm{ng} \mathrm{mL}^{-1}$ for AA II. Our developed UHPLC-MS/MS shows advantages in both the analytical time and sensitivity. Jacob et al. used UHPLC-MS for the detection of AAs [34]. Their analytical time is close to our study. They used the chemometrics approach for AAs detection. Since quantitation work was not included in their study, they did not evaluate the accuracy and matrix effect of their system. They also did not optimize their conditions for the best sensitivity. The applicability of their method to quantitatively analyze AAs in herbal products and biological samples

Table 4

Pharmacokinetic parameters after single oral administration of $10 \mathrm{mg} / \mathrm{kg}$ standard in female Wister rats.

\begin{tabular}{lcc}
\hline & AAI & AAII \\
\hline$T_{\max }(\min )$ & 30 & 45.0 \\
$\left.C_{\max }(\mathrm{ng} \mathrm{mL})^{-1}\right)$ & 7249.3 & 77.7 \\
AUC $\left.(\mathrm{ng} \mathrm{mL})^{-1} \mathrm{~min}\right)$ & $716,936.3$ & $31,344.8$ \\
$T_{1 / 2}(\mathrm{~min})$ & 234.6 & 800.7 \\
$k\left(\mathrm{~min}^{-1}\right)$ & 0.003 & 0.0009 \\
Oral clearance $\left(\mathrm{mL} \mathrm{min}^{-1}\right)$ & 2.9 & 66.0 \\
\hline
\end{tabular}

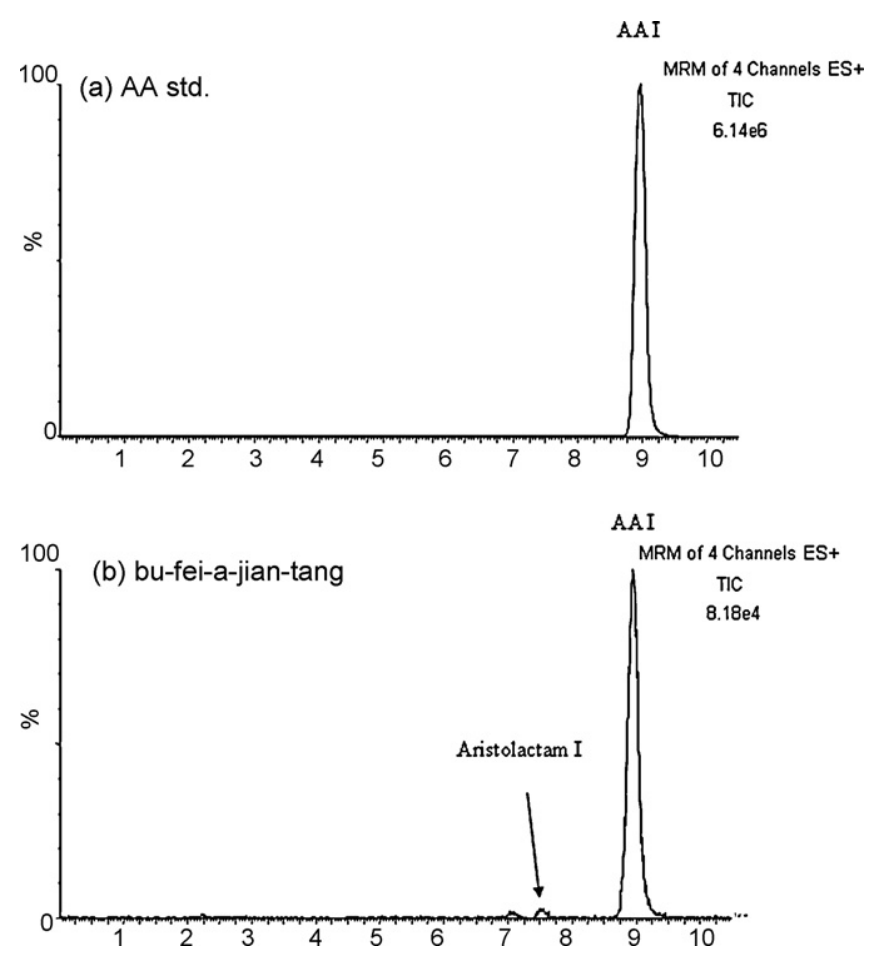

Fig. 7. UHPLC-MS/MS chromatogram (MRM) of liver microsome treated with (a) AA I standard; (b) Bu-fei-a-jiau-tang for $30 \mathrm{~min}$. UHPLC-MS/MS conditions are the same with that described in Fig. 3.

was unknown. Comparing to their method, the high selectivity of the presented UHPLC-MS/MS conditions significantly minimized the matrix effect, which allows this method to be applied to analyze complex samples. Recently, capillary electrophoresis and HPLC coupled with fluorescence detector have been used for sensitive detection of AAs in herbal products $[10,19,22]$. Although the LODs could reach the sub-ppb levels when coupling with fluorescence detection, chemical reaction should be preformed before analysis. The proposed UHPLC-MS/MS method is superior to those methods in its simplicity in sample preparation.

\section{Conclusion}

A fast, sensitive and reliable UHPLC-MS/MS method for the determination of AA I and AA II was established in this study. By using a mixture of $10 \mathrm{mM}$ ammonium formate buffer ( $\mathrm{pH}$ 3.0) and acetonitrile as mobile phase with gradient elution, AAs could be determined within $10 \mathrm{~min}$. The LODs was $0.14 \mathrm{ng} \mathrm{mL}^{-1}$ for AA I and $0.26 \mathrm{ng} \mathrm{mL}^{-1}$ for AA II. Due to its high selectivity and sensitivity, the developed method could be directly used to analyze crude herbal extracts as well as biological samples without solid phase extraction or liquid/liquid extractions. The applicability of the method was demonstrated by applying the developed method to analyze various herbal products, rat serum and liver microsome incubation samples. The results revealed that the developed approach is powerful for quality control of herbal products and pharmacokinetic study of AAs.

\section{Acknowledgements}

This study was supported by a research grant (CCMP96-RD-044) from the Committee on Chinese Medicine and Pharmacy, Department of Health, Executive Yuan, Taiwan. We thank Waters Asia Ltd., Taiwan Branch for technical support. We also acknowledge Dr. Shao-Wen Sun for editing this manuscript. 


\section{References}

[1] K. Hashimoto, M. Higuchi, B. Makino, I. Sakakibara, M. Kubo, Y. Komatsu, M. Maruno, M. Okada, J. Ethnopharmacol. 64 (1999) 185-189.

[2] M.Y. Shen, C.L. Liu, G. Hsiao, C.Y. Liu, K.H. Lin, D.S. Chou, J.R. Sheu, Planta Med. 74 (2008) 1240-1245.

[3] J.J. Moreno, Immunopharmacology 26 (1993) 1-9.

[4] B.S. Vishwanath, A.A. Fawzy, R.C. Franson, Inflammation 12 (1988) 549-561.

[5] J.L. Vanherweghem, M. Depierreux, C. Tielemans, D. Abramowicz, M. Dratwa, M. Jadoul, C. Richard, D. Vandervelde, D. Verbeelen, R. Vanhaelen-Fastre, et al., Lancet 341 (1993) 387-391.

[6] J.L. Nortier, M.C. Martinez, H.H. Schmeiser, V.M. Arlt, C.A. Bieler, M. Petein, M.F. Depierreux, L. De Pauw, D. Abramowicz, P. Vereerstraeten, J.L. Vanherweghem, N. Engl. J. Med. 342 (2000) 1686-1692.

[7] Available: U.S. Food and Drug Administration, Dietary supplements: aristolochic acid, http://www.cfsan.fda.gov/ dms/ds-bot.html.

[8] L.S. Gold, T.H. Slone, N. Engl. J. Med. 349 (2003) 1576-1577.

[9] F.D. Debelle, J.L. Vanherweghem, J.L. Nortier, Kidney Int. 74 (2008) 158169.

[10] S.C. Hsieh, M.F. Huang, B.S. Lin, H.T. Chang, J. Chromatogr. A 1105 (2006) 127-134.

[11] M.L. Pailer, L. Belohlav, E. Simonitsch, Angew. Chem. 68 (1955) 386.

[12] V.M. Arlt, M. Stiborova, H.H. Schmeiser, Mutagenesis 17 (2002) 265-277.

[13] J.R. Ioset, G.E. Raoelison, K. Hostettmann, Food Chem. Toxicol. 41 (2003) 29-36.

[14] W. Chan, K.M. Hui, W.T. Poon, K.C. Lee, Z. Cai, Anal. Chim. Acta 576 (2006) $112-116$.

[15] L. Wei, R.K. Li, T. Bo, H.W. Liu, X.F. Feng, S.L. Hu, Chromatographia 59 (2004) 233-236.

[16] J. Yuan, L. Nie, D. Zeng, X. Luo, F. Tang, L. Ding, Q. Liu, M. Guo, S. Yao, Talanta 73 (2007) 644-650.

[17] W. Li, S. Gong, D. Wen, B. Che, Y. Liao, H. Liu, X. Feng, S. Hu, J. Chromatogr. A 1049 (2004) 211-217.

[18] W. Li, Z. Chen, Y. Liao, H. Liu, Electrophoresis 27 (2006) 837-841.

[19] J. Yuan, Q. Liu, W. Zhu, L. Ding, F. Tang, S. Yao, J Chromatogr. A 1182 (2008) 85-92.

[20] H. Yue, W. Chan, L. Guo, Z. Cai, J. Chromatogr. B 877 (2009) 995-999.
[21] J. Yuan, X. Luo, M. Guo, J. Wu, W. Yang, R. Yu, S. Yao, Talanta 78 (2009) 1141-1147.

[22] X. Zhou, C. Zheng, J. Sun, T. You, J. Chromatogr. A 1109 (2006) 152-159.

[23] F. Wei, Y.Q. Feng, Talanta 74 (2008) 619-624.

[24] G.C. Kite, M.A. Yule, C. Leon, M.S. Simmonds, Rapid Commun. Mass Spectrom. 16 (2002) 585-590.

[25] M.C. Lee, C.H. Tsao, S.C. Iou, W.C. Chuang, S.J. Sheu, J. Sep. Sci. 26(2003) 818-822

[26] S.A. Chan, M.J. Chen, T.Y. Liu, M.R. Fuh, J.F. Deng, M.L. Wu, S.J. Hsieh, Talanta 60 (2003) 679-685.

[27] C.Y. Huang, M.C. Tseng, J.H. Lin, J. Food Drug Anal. 13 (2005) 125-131.

[28] J. Yuan, Q. Liu, G. Wei, F. Tang, L. Ding, S. Yao, Rapid Commun. Mass Spectrom. 21 (2007) 2332-2342.

[29] T.T. Jong, M.R. Lee, S.S. Hsiao, J.L. Hsai, T.S. Wu, S.T. Chiang, S.Q. Cai, J. Pharm. Biomed. Anal. 33 (2003) 831-837.

[30] B.K. Matuszewski, M.L. Constanzer, C.M. Chavez-Eng, Anal. Chem. 70 (1998) 882-889.

[31] B.K. Matuszewski, M.L. Constanzer, C.M. Chavez-Eng, Anal. Chem. 75 (2003) 3019-3030.

[32] L. Novakova, L. Matysova, P. Solich, Talanta 68 (2006) 908-918.

[33] S.A. Wren, P. Tchelitcheff, J. Chromatogr. A 1119 (2006) 140-146.

[34] J.M. Cunliffe, S.B. Adams-Hall, T.D. Maloney, J. Sep. Sci. 30 (2007) 1214-1223.

[35] J.C. Van De Steene, W.E. Lambert, J. Am. Soc. Mass Spectrom. 19(2008) 713-718.

[36] S.S. Jacob, N.W. Smith, C. Legido-Quigley, J. Sep. Sci. 30 (2007) 1200-1206.

[37] S.M. Chen, M.Y. Fan, C.C. Tseng, Y. Ho, K.Y. Hsu, Toxicon 50 (2007) 180-188.

[38] T.H. Tsai, C.J. Chou, L.C. Lin, W.J. Tsai, C.F. Chen, J. Liq. Chromatogr. 16 (1993) 1173-1182.

[39] Y. Xiao, M. Ge, X. Xue, C. Wang, H. Wang, X. Wu, L. Li, L. Liu, X. Qi, Y. Zhang, Y Li, H. Luo, T. Xie, J. Gu, J. Ren, Kidney Int. 73 (2008) 1231-1239.

[40] M. Stiborova, B. Sopko, P. Hodek, E. Frei, H.H. Schmeiser, J. Hudecek, Cancer Lett 229 (2005) 193-204.

[41] X. Fu, Y. Liu, W. Li, Y. Liao, M. Zhai, H. Liu, Proceedings of the 22nd International Symposium on Microscale Bioseparations and Methods for Systems Biology, 2008.

[42] J.S. Page, R.T. Kelly, K. Tang, R.D. Smith, J. Am. Soc. Mass Spectrom. 18 (2007) 1582-1590.

[43] H.F. Kasai, M. Tsubuki, S. Matsuo, T. Honda, Rapid Commun. Mass Spectrom. 21 (2007) 1100-1114. 